\title{
Numerical investigation of the velocity field and separation efficiency of deoiling hydrocyclones
}

\author{
MADDAHIAN Reza*, ASADI Mohammad and FARHANIEH Bijan \\ Center of Excellence in Energy Conversion, School of Mechanical Engineering, Sharif University of Technology, Tehran, Iran
}

(C) China University of Petroleum (Beijing) and Springer-Verlag Berlin Heidelberg 2012

\begin{abstract}
Three-dimensional simulation of a multiphase flow is performed using the EulerianEulerian finite volume method in order to evaluate the separation efficiency and velocity field of deoiling hydrocyclones. The solution is developed using a mass conservation-based algorithm (MCBA) with collocated grid arrangement. The mixture approach of the Reynolds stress model is also employed in order to capture features of turbulent multiphase swirling flow. The velocity field and separation efficiency of two different configurations of deoiling hydrocyclones are compared with available experimental data. The comparison shows that the separation efficiency can be predicted with high accuracy using computational fluid dynamics. The velocity fields are also in good agreement with available experimental velocity measurements. Special attention is drawn to swirl intensity in deoiling hydrocyclones and it is shown that the differences in velocity and volume fraction fields of different configurations are related to swirl distribution.
\end{abstract}

Key words: Deoiling hydrocyclone, numerical simulation, Eulerian-Eulerian approach, swirl intensity

\section{Introduction}

Having an efficient and reliable system for oil-water separation especially in the offshore oil and gas industry is of crucial importance. Due to platform movement and space, weight and operating limitations in offshore industries, using common methods (e.g. gravity based vessels), for oilwater separation is ineffective. On the other hand, producing oil on offshore platforms is often accompanied by large discharge of oil-contaminated water into the sea, resulting in environmental pollution.

Therefore, there is a need for high efficiency compact separators capable of operation in various operating conditions. One solution to the mentioned problem is the use of hydrocyclone separators. The advantages of hydrocyclones, compared to other methods, are those of simple design, easy installation and operation, lack of moving parts, low manufacturing and maintenance costs. The hydrocyclones are therefore an economical and effective way for produced water treatment (Van den Broek et al, 1991; Van den Broek and Plat, 1998)

The main differences between the separation mechanisms of deoiling hydrocyclones and that of desanders are described below (Thew, 1986; Caldentey, 2000):

- The density difference in liquid-liquid mixtures encountered in deoiling hydrocyclones is smaller than solidliquid types present in desanders. Therefore, separation of two immiscible liquids takes much more effort than separation of solid from liquid.

*Corresponding author. email: r_maddahian@mech.sharif.edu Received October 18, 2011
- The liquid droplets tolerate lower shear rates compared to solid particles. Large droplets break down into smaller ones whenever the shear rate increases to a critical level. The separation process for small liquid droplets is more difficult than larger ones. On the other hand, if two droplets were close enough, they might coalesce.

- Due to intrinsic differences in flow splits of desander and deoiling hydrocyclones, the flow features of the continuous phase is not the same.

- The centrifugal force makes solid particles migrate to the wall region in desander hydrocyclones while making oil droplets move to the center in the deoiling type. So the nearwall region is of high importance in desander hydrocyclones as opposed to the center flow features in the deoiling type.

The use of common hydrocyclones for oil-water separation was first suggested by Simkin and Olney (1956) and Sheng et al (1974), but fundamental studies of deoiling hydrocyclones was started from 1980 by Colman and Thew. Several researchers (Colman et al, 1980; Colman, 1981; Colman and Thew, 1980; 1983; 1988) performed experiments to study deoiling hydrocyclones. Experimental results showed that the separation efficiency of hydrocyclones is independent of the flow split if the flow split is between 0.5 to 10 percent. Moreover, the size distribution in the outlet is independent of the flow split for constant droplet size distribution in the inlet. The migration probability curves are also independent of the flow split. The problems of using hydrocyclones for water treatment were investigated by Thew (1986) and a new design was proposed. The new design had specific advantages such as high swirl flow, smaller size preventing large pressure drop and minimum instability and turbulence near the axis. 
The operational curves, principle of operations and the first field study of hydrocyclones were presented by Meldrum (1987), after which several field tests of hydrocyclones were conducted by various researchers (Van den Broek and Plat, 1991; Van den Broek et al, 1998; Choi, 1990; Noort et al, 1990; Falnigan et al, 1989; Jones, 1993; Ditria and Hoyack, 1994).

The first attempt on optimizing hydrocyclones was conducted by Young et al (1994). They measured the flow behavior in a 35-mm hydrocyclone, designed by Colman and Thew (1980), and then compared the results with a newly modified design. They studied the effects of operational variables and geometrical parameters, such as inlet size, cylindrical diameter, cone angle, straight section length, flow rate, and droplet diameter on the separation efficiency. Based on their experimental results, a new geometry was proposed for hydrocyclones.

Recent investigations on hydrocyclones focus on operational parameters (Belaidi and Thew, 2003; Husveg et al, 2007), velocity field (Bai et al, 2009) and distribution of oil droplets (Zhou et al, 2010) in deoiling hydrocyclones.

Due to the difficulty of numerical simulations of liquid-liquid hydrocyclones, the majority of the studies focused on experimental investigations with only few studies concentrating on numerical simulations of deoiling hydrocyclones.

Hargreaves and Silvester (1990) simulated the oil-water flow in deoiling hydrocyclones using the Eulerian-Lagrangian approach. They used the algebraic stress model to simulate the flow in a $2 \mathrm{D}$ cylindrical coordinate system. In the dispersed phase, they ignored the effect of particle-particle interaction, slip and droplet coalescence. The obtained results were in acceptable agreement with experimental data. The flow field, velocity distribution and separation efficiency of a $10-\mathrm{mm}$ deoiling hydrocyclone was obtained by Grady et al (2003) using the algebraic slip mixture (ASM) multiphase model. In order to simulate the high swirling flow (swirl number 8.4) the Reynolds stress model (RSM) was used.

Simulation of miniature hydrocyclones for downhole separation was conducted by Petty and Park (2004). Direct numerical simulation showed that the $3 g$ centrifugal acceleration was created in a $5-\mathrm{mm}$ miniature hydrocyclones for estimated pressure drop and flow rates of $1 \mathrm{~L} / \mathrm{s}$ and $1 \mathrm{kPa}$, respectively. Huang (2005) simulated the three dimensional turbulent flow in deoiling hydrocyclones using the EulerianEulerian approach and the Reynolds stress model. Results showed accumulation of oil near the axis. The separation efficiency was also determined based on phase concentration. The separation curve for Colman type hydrocyclones was in good agreement with measured ones. It must be mentioned that no velocity distribution was reported by Huang. Noroozi and Hashemabadi $(2009 ; 2011)$ investigated the effect of various inlet types and inlet chamber body profiles on the separation efficiency of deoiling hydrocyclones. The separation efficiency was improved $10 \%$ and $8 \%$ with the use of helical inlet and exponential body profile, respectively. Kharoua et al (2010) conducted a complete review of hydrocyclones used for deoiling purposes.
The literature review showed that nearly all conducted numerical studies done on deoiling hydrocyclones have mainly focused on separation efficiency of deoiling hydrocyclones. In order to predict the separation efficiency, the velocity field for both phases should be obtained accurately. In other words, the more precise the determination of the velocity field, the more accurate the separation efficiency.

The aim of this research is to introduce an appropriate multiphase model and demonstrate the capability of computational fluid dynamics in predicting separation efficiency, oil droplet distribution and the velocity field of multiphase flow using the general Eulerian-Eulerian multiphase model. The results also show the importance of swirl intensity in designing hydrocyclones. It should be mentioned that previous numerical investigations (Grady et al, 2003; Noroozi and Hashemabadi, 2009; 2011) considered the simple algebraic slip mixture model which could not correctly show the distribution of oil droplets in hydrocyclones. Optimization of hydrocyclones is not taken into consideration in this research and will be taken into account in future work.

\section{Mathematical model}

A multiphase flow generally contains dispersed particles and droplets. The modeling of instantaneous governing equations for the continuous phase and each particle is computationally intensive except for the case of ideal flow at low Reynolds numbers. Therefore, the local instantaneous equations should be averaged using appropriate operators (Ishii, 1975; Drew, 1983). The following governing equations derived based on ensemble-averaging of Navier-Stokes equations for each phase using the Favre-weighting method. Details of the averaging procedure and assumptions can be found in (Drew, 1983; Enwald et al, 1996).

\subsection{Governing equations}

Eulerian-Eulerian or two-fluid transport equations (two continuity and six momentum equations) are as follows (Enwald et al, 1996):

$$
\begin{aligned}
& \frac{\partial\left(\alpha^{(k)} \rho^{(k)}\right)}{\partial t}+\nabla \cdot\left(\alpha^{(k)} \rho^{(k)} \boldsymbol{U}^{(k)}\right)=0 \\
& \frac{\partial\left(\alpha^{(k)} \rho^{(k)} \boldsymbol{U}^{(k)}\right)}{\partial t}+\nabla \cdot\left(\alpha^{(k)} \rho^{(k)} \boldsymbol{U}^{(k)} \boldsymbol{U}^{(k)}\right) \\
& =\nabla \cdot \overline{\bar{\tau}}^{(k)}+\alpha^{(k)}\left(-\nabla p+B^{(k)}\right)+\boldsymbol{I}_{M}^{(k)}+\boldsymbol{F}^{(k)}
\end{aligned}
$$

where $\alpha^{(k)}$ stands for the volume fraction of phase $(k) ; \rho^{(k)}$ is the density of phase $(k), \mathrm{kg} / \mathrm{m}^{3} ; U^{(k)}$ denotes the averaged velocity of phase $(k), \mathrm{m} / \mathrm{s} ; \overline{\bar{\tau}}^{(k)}, p$ and $B^{(k)}$ are the stress tensor $\left(\mathrm{N} / \mathrm{m}^{2}\right)$, pressure shared by all phases $\left(\mathrm{kg} /\left(\mathrm{m} \cdot \mathrm{s}^{2}\right)\right)$ and the body forces per unit volume of phase $(k)\left(\mathrm{kg} /\left(\mathrm{m}^{2} \cdot \mathrm{s}^{2}\right)\right)$, respectively; $\boldsymbol{F}^{(k)}$ the term includes all other forces such as lift and virtual mass, $\left(\mathrm{kg} /\left(\mathrm{m}^{2} \cdot \mathrm{s}^{2}\right)\right)$.

$\boldsymbol{I}_{M}^{(k)}$ is the momentum transfer to phase $(k)$ due to phase interaction and can be written as: 


$$
\boldsymbol{I}_{M}^{(k)}=\sum_{m \neq k} g^{(k m)}\left[\left(\boldsymbol{U}^{(m)}-\boldsymbol{U}^{(k)}\right)-\left(\frac{\overline{\alpha^{(m)} \boldsymbol{u}^{(m)}}}{\overline{\alpha^{(m)}}}-\frac{\overline{\alpha^{(k)} \boldsymbol{u}^{\prime(k)}}}{\overline{\alpha^{(k)}}}\right)\right](3) \quad \tilde{\boldsymbol{\varepsilon}}_{i j}=\frac{2}{3} \tilde{\rho} \tilde{\boldsymbol{\varepsilon}} \boldsymbol{\delta}_{i j}
$$

The pressure strain term, often referred as pressure-strain redistribution term $\left(\Pi_{i j}\right)$, is decomposed into the rapid and slow pressure strain terms and modeled according to (Wilcox, 1994):

$$
\begin{aligned}
\tilde{\Pi}_{i j}= & -C_{1} \frac{\tilde{\varepsilon}}{\tilde{k}}\left(\tilde{\boldsymbol{\tau}}_{i j}+\frac{2}{3} \tilde{\rho} \tilde{k} \boldsymbol{\delta}_{i j}\right)-\hat{\lambda}\left(\tilde{\boldsymbol{P}}_{i j}-\frac{2}{3} \tilde{P} \boldsymbol{\delta}_{i j}\right) \\
& -\hat{\beta}\left(\tilde{\boldsymbol{D}}_{i j}-\frac{2}{3} \tilde{P} \boldsymbol{\delta}_{i j}\right)-\hat{\gamma} \tilde{\rho} \tilde{k}\left(\tilde{\boldsymbol{S}}_{i j}-\frac{1}{3} \tilde{S}_{k k} \boldsymbol{\delta}_{i j}\right) \\
& +\left[0.125 \frac{\tilde{\varepsilon}}{\tilde{k}}\left(\tilde{\boldsymbol{\tau}}_{i j}+\frac{2}{3} \tilde{\rho} \tilde{k} \boldsymbol{\delta}_{i j}\right)-0.015\left(\tilde{\boldsymbol{P}}_{i j}-\tilde{\boldsymbol{D}}_{i j}\right)\right] \frac{\tilde{k}^{3 / 2}}{\tilde{\varepsilon} n}
\end{aligned}
$$

In case the last term in Eq. (8) is modeled as suggested in LRR, the simulations show numerical instabilities. So the mixture Reynolds stress tensor diffusion is simplified as follows (Lien and Leschziner, 1994):

$$
\frac{\partial}{\partial x_{k}}\left[\tilde{v} \frac{\partial \tilde{\boldsymbol{\tau}}_{i j}}{\partial x_{k}}+\tilde{\boldsymbol{C}}_{i j k}\right]=\frac{\partial}{\partial x_{k}}\left[\left(\tilde{v}+\frac{\tilde{v}_{T}}{\sigma_{k}}\right) \frac{\partial \tilde{\tau}_{i j}}{\partial x_{k}}\right]
$$

Constants, equations and auxiliary relations for the LRR model are defined as follows:

Dissipation rate equation (Wilcox, 1994):

$$
\begin{aligned}
& \frac{\partial(\tilde{\rho} \tilde{\varepsilon})}{\partial t}+\frac{\partial}{\partial x_{k}}\left(\tilde{\rho} \tilde{\boldsymbol{U}}_{k} \tilde{\varepsilon}\right) \\
& =C_{\varepsilon 1} \frac{\tilde{\varepsilon}}{\tilde{k}} \tilde{\boldsymbol{\tau}}_{i k} \frac{\partial \tilde{\boldsymbol{U}}_{i}}{\partial x_{k}}-C_{\varepsilon 2} \tilde{\rho} \frac{\tilde{\varepsilon}^{2}}{\tilde{k}}-C_{\varepsilon} \frac{\partial}{\partial x_{k}}\left[\frac{\tilde{k}}{\tilde{\varepsilon}} \tilde{\boldsymbol{\tau}}_{k i} \frac{\partial \tilde{\varepsilon}}{\partial x_{i}}\right]
\end{aligned}
$$

mixture.

\subsection{Turbulence model}

In order to simulate the complex swirling flow, employing an appropriate turbulence model with high resolution scheme for discretizing governing equations plays a key role in having acceptable and accurate results. Previous numerical simulations of the swirling flow showed applicability of the Reynolds stress model (RSM) for simulating such flow inside the hydrocyclones (Grady et al, 2003; Huang, 2005). So the mixture approach of RSM Launder-Reece-Rodi (RSM-LRR) (Launder et al, 1975) model is adopted in this work.

The transport equation for the Reynolds stress tensor $\left(\tilde{\tau}_{i j}\right)$ of the mixture is as follows (Wilcox, 1994):

$$
\frac{\partial\left(\tilde{\boldsymbol{\tau}}_{i j}\right)}{\partial t}+\frac{\partial}{\partial x_{k}}\left(\tilde{\boldsymbol{U}}_{k} \tilde{\boldsymbol{\tau}}_{i j}\right)=\tilde{\boldsymbol{P}}_{i j}-\tilde{\boldsymbol{\varepsilon}}_{i j}+\tilde{\Pi}_{i j}+\frac{\partial}{\partial x_{k}}\left[\tilde{\boldsymbol{v}} \frac{\partial \tilde{\boldsymbol{\tau}}_{i j}}{\partial x_{k}}+\tilde{\boldsymbol{C}}_{i j k}\right] \text { (8) }
$$

where $\tilde{\boldsymbol{P}}_{i j}$ is the stress production and defined by the following equation (Wilcox, 1994):

$$
\tilde{\boldsymbol{P}}_{i j}=-\left(\tilde{\boldsymbol{\tau}}_{i k} \frac{\partial \tilde{\boldsymbol{U}}_{j}}{\partial x_{k}}+\tilde{\boldsymbol{\tau}}_{j k} \frac{\partial \tilde{\boldsymbol{U}}_{i}}{\partial x_{k}}\right)
$$

Using Kolmogorov's hypothesis of local isotropy, $\tilde{\varepsilon}_{i j}$ can be modeled as (Wilcox, 1994):
Auxiliary relations (Wilcox, 1994):

$$
\left\{\begin{array}{l}
\tilde{\boldsymbol{D}}_{i j}=\tilde{\boldsymbol{\tau}}_{i k} \frac{\partial \tilde{\boldsymbol{U}}_{k}}{\partial x_{j}}+\tilde{\tau}_{j k} \frac{\partial \tilde{\boldsymbol{U}}_{k}}{\partial x_{i}} \\
\tilde{P}=\frac{1}{2} \tilde{P}_{k k}
\end{array}\right.
$$

Closure coefficient (Wilcox, 1994):

$$
\begin{array}{lll}
\hat{\lambda}=\left(8+C_{2}\right) / 11 & \hat{\beta}=\left(8 C_{2}-2\right) / 11 & \hat{\gamma}=\left(60 C_{2}-4\right) / 55 \\
C_{1}=1.8 & C_{2}=0.6 & \sigma_{k}=0.82 \\
C_{\varepsilon}=0.18 & C_{\varepsilon 1}=1.44 & C_{\varepsilon 2}=1.92
\end{array}
$$

As it can be seen all of the variables in Eqs. (8) to (15) are averaged. The averaging operator for physical properties and other quantities are defined in Eqs. (16) and (17) respectively.

$$
\tilde{\varphi}=\sum_{k=1}^{m} \alpha^{(k)} \varphi^{(k)}
$$




$$
\tilde{\varphi}=\frac{\sum_{k=1}^{m} \alpha^{(k)} \rho^{(k)} \varphi^{(k)}}{\sum_{k=1}^{m} \alpha^{(k)} \rho^{(k)}}
$$

\section{Numerical method}

\subsection{Geometry of the problem and mesh generation}

Two different geometries used for numerical modeling of deoiling hydrocyclones are shown in Fig. 1. The numerical results of the separation efficiency and velocity distribution are obtained and compared to available experimental measurements. The geometrical parameters of these configurations are shown in Table 1.

Three non-uniform structured grids are used to show the grid independency of the results. Nodal distributions in both geometries for coarse, medium and fine grids are shown in Table 2.

As shown in Fig. 2, the maximum differences in the tangential velocity in the radial direction, located at $z / D=2.0(z$ is the axial distance from the top wall, $\mathrm{mm}$ ),

- between the coarse and medium grids of case 1 and case 2 are $5 \%$ and $7 \%$, respectively

- between the medium and fine grids of case 1 and case 2 are $1 \%$ and $1 \%$, respectively.

Therefore, the medium grid is selected for numerical simulation in order to reduce the computational costs. The generated mesh for simulating the flow inside deoiling hydrocyclones is shown in Fig. 3. It can be seen that the mesh becomes finer in regions with higher gradient especially near the wall and the core of hydrocyclone.

Table 1 Geometrical parameters of deoiling hydrocyclones

\begin{tabular}{|c|c|c|c|c|c|c|c|c|c|c|}
\hline Case & $\begin{array}{l}\text { Hydrocyclone } \\
\text { diameter } \\
D, \mathrm{~m}\end{array}$ & $D_{\mathrm{n}}, \mathrm{m}$ & $L_{\mathrm{c}} / D$ & $L_{\mathrm{u}} / D$ & $L_{\sqrt{ }} / D$ & $D_{\mathrm{d}} / D$ & $D_{\mathrm{u}} / D$ & $D_{\mathrm{i}} / D$ & $\alpha$ & $\beta$ \\
\hline Case 1 (Young et al, 1994) & 0.07 & 0.035 & 1.0 & 13.0 & - & 0.042 & 0.363 & 0.226 & 20 & 1.5 \\
\hline Case 2 (Bai et al, 2009) & 0.035 & - & 1.0 & 9.71 & 0.1 & 0.07 & 0.23 & 0.322 & 6 & - \\
\hline
\end{tabular}

Notes: $D$ is the hydrocyclone diameter; $D_{\mathrm{n}}$ is the characteristic diameter of the hydrocyclone; $L_{\mathrm{c}}, L_{\mathrm{u}}$, and $L_{\mathrm{v}}$ are the lengths of the cylindrical section, underflow, and the vortex finder, respectively; $D_{\mathrm{o}}, D_{\mathrm{u}}$, and $D_{\mathrm{i}}$ are the diameters of overflow, underflow and the inlet, respectively; $\alpha$ and $\beta$ are the first and second cone angle of hydrocyclone.

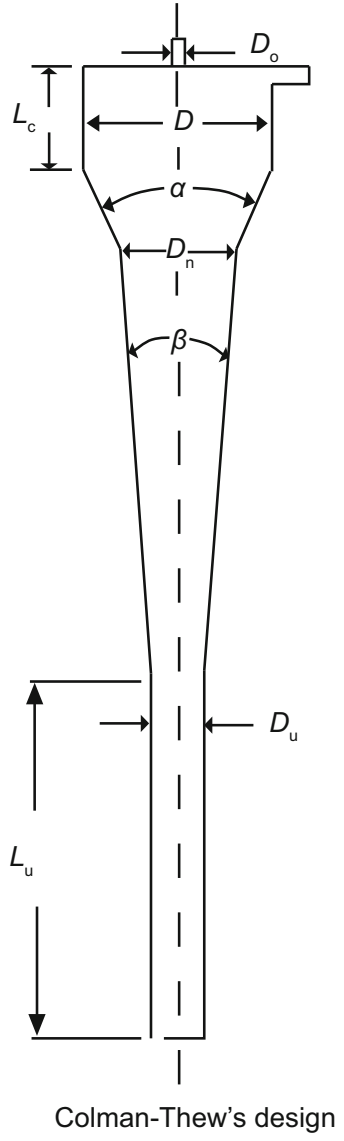

(a) Case 1
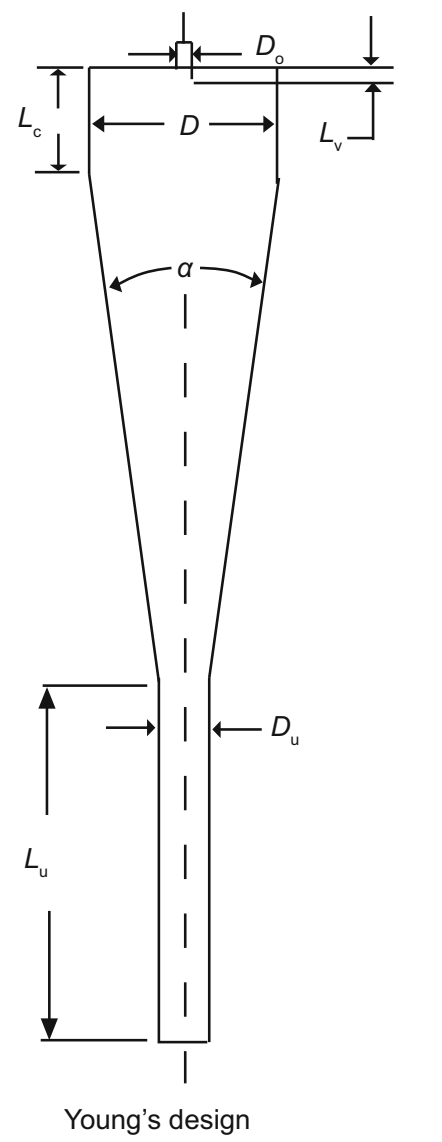

(b) Case 2

Fig. 1 Two different configurations of deoiling hydrocyclones

Table 2 The nodal distributions of hydrocyclone mesh

\begin{tabular}{cccc}
\hline \multirow{2}{*}{ Case } & \multicolumn{3}{c}{ Nodal distribution $(r, \theta, z)$} \\
\cline { 2 - 4 } & Coarse & Medium & Fine \\
\hline Case 1 & $20 \times 48 \times 260$ & $25 \times 60 \times 300$ & $30 \times 64 \times 400$ \\
Case 2 & $20 \times 48 \times 200$ & $25 \times 60 \times 250$ & $30 \times 64 \times 300$ \\
\hline
\end{tabular}

\subsection{Boundary conditions}

There are three types of boundaries (inlet, outlet and wall) considered as follows: Inlet

All of the variables are known in this region. Uniform velocity and volume fraction with turbulence intensity of $5 \%$ are employed.

Wall

No slip condition $\left(\boldsymbol{U}^{(k)}=0\right)$ is assumed on the walls. Turbulence quantities near the wall are handled by the standard wall function.

Outlet

The gauge static pressure, determined based on the desired split ratio, is assumed to be fixed at the minimum radius of each outlet, i.e. the overflow and underflow. Velocities are calculated using local continuity equation for boundary cells (Ferziger and Peric, 2002).

Operational parameters for both designs are shown in Table 3.

\subsection{Solution methodology}

For numerical investigation of the flow field inside 


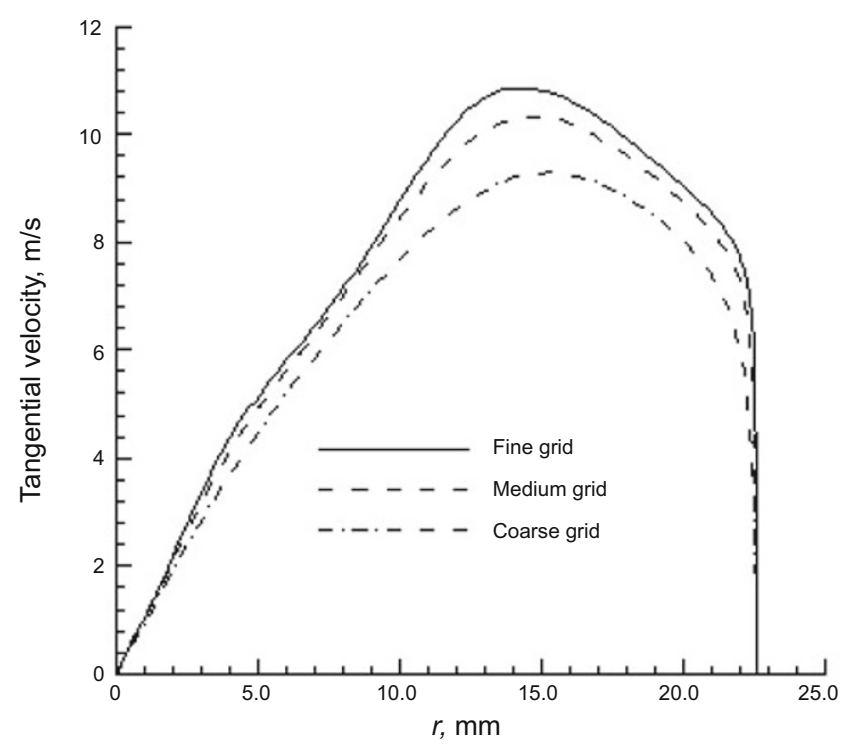

(a) Case 1

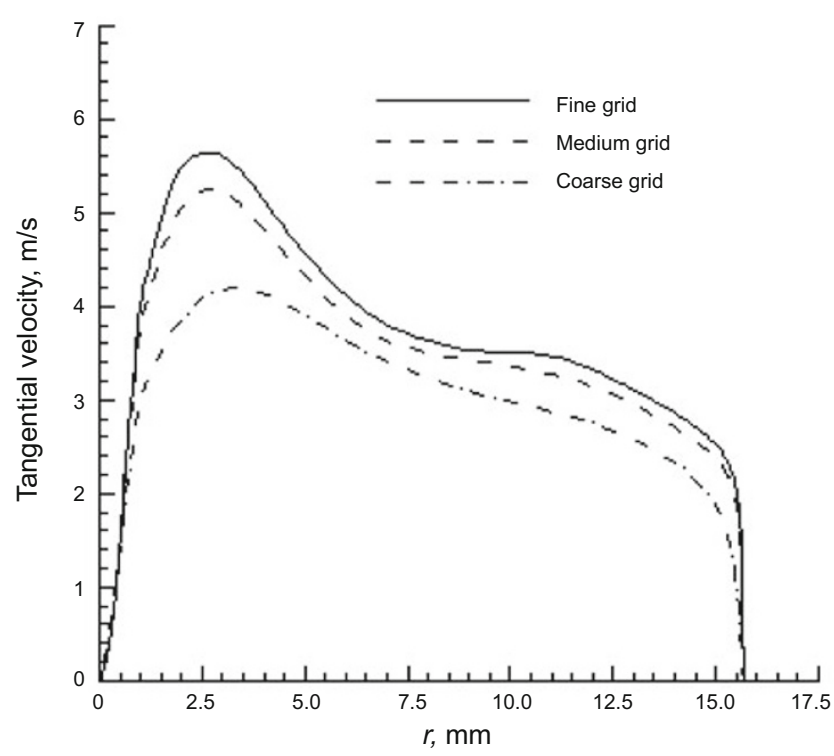

(b) Case 2

Fig. 2 Variation of the tangential velocity in $z / D=2.0$ for different grids deoiling hydrocyclones a new finite volume code, EULERCALC, has been developed based on CALC-BFC (Davidson and Farhanieh, 1991). The solution algorithm of multiphase flow is developed using mass conservation-based algorithms (MCBA) method, proposed by Moukalled and Darwish (2004). The steps of MCBA are as follows:
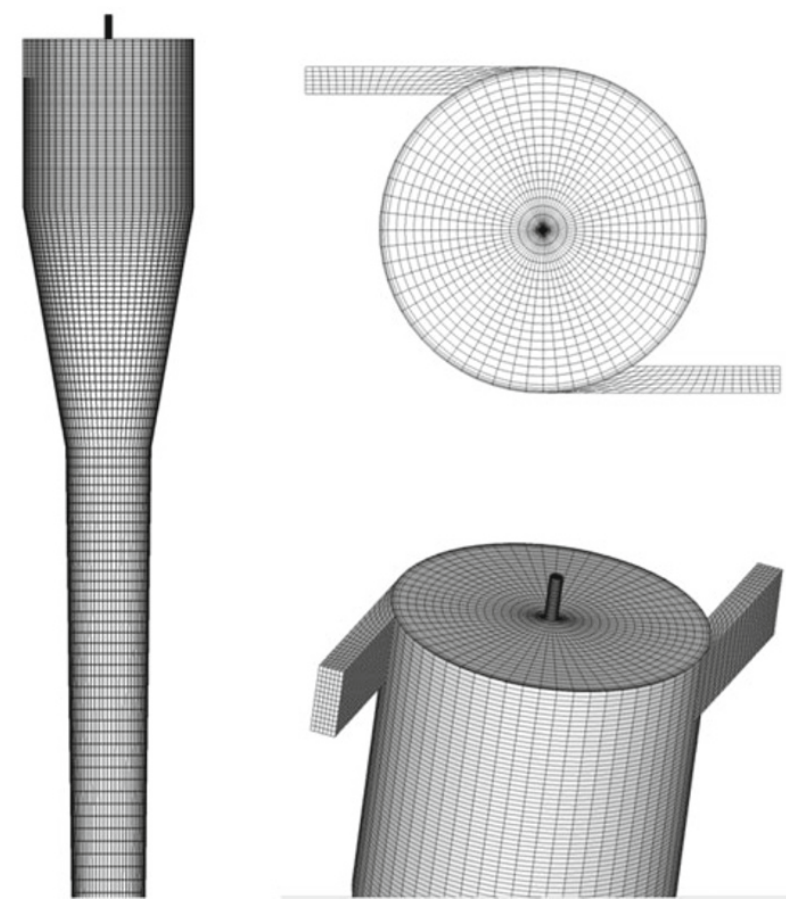

Fig. 3 Generated mesh for hydrocyclone simulations

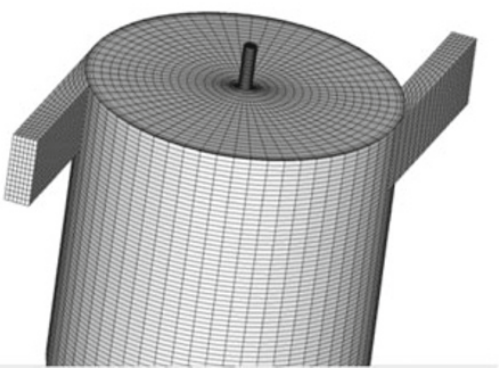

- Solve momentum equations for each phase to obtain velocities;

- Solve pressure correction equation derived based on global conservation of mass;

- Correct velocity of each phase;

- Correct pressure;

- Calculate the averaged quantities and solve Reynolds stress transport equation of mixture;

- Solve continuity of each phase to obtain the volume fractions.

The above steps are repeated until convergence. For velocity calculations on the faces of the control volumes, the Rhie-Chow (Rhie and Chow, 1983) interpolation method is used and the modification of SIMPLEC for multifluid systems (Darwish et al, 2001) handles the linkage between the velocities and pressure.

The partial elimination algorithm (PEA) (Darwish et al, 2001) is used to reduce the linkage between phases and accelerate the convergence. The global continuity equation, used for calculating shared pressure between phases, is normalized using $\left(\rho^{(k)}\right)$ as a weighting factor in order to reduce the continuity error. The convergence rate is accelerated by solving two implicit volume fractions and then

Table 3 Operational parameters of deoiling hydrocyclones

\begin{tabular}{ccccccccc}
\hline Case & $\begin{array}{c}Q_{\mathrm{i}} \\
\mathrm{m}^{3} / \mathrm{h}\end{array}$ & $R e_{\mathrm{i}}$ & $\begin{array}{c}R \\
\%\end{array}$ & $\alpha_{\mathrm{i}}$ & $\begin{array}{c}\rho_{\text {water }} \\
\mathrm{kg} / \mathrm{m}^{3}\end{array}$ & $\begin{array}{c}\rho_{\text {oil }} \\
\mathrm{kg} / \mathrm{m}^{3}\end{array}$ & $\begin{array}{c}\mu_{\text {water }} \\
\mathrm{kg} /(\mathrm{m} \cdot \mathrm{s})\end{array}$ & $\begin{array}{c}\mu_{\text {oil }} \\
\mathrm{kg} /(\mathrm{m} \cdot \mathrm{s})\end{array}$ \\
\hline Case 1 (Young et al, 1994) & 4.84 & $5.9 \times 10^{4}$ & $0.5-10$ & 0.05 & & & $\begin{array}{c}d \\
\mu \mathrm{m}\end{array}$ \\
Case 2 (Bai et al, 2009) & 1.5 & $2.8 \times 10^{4}$ & 5 & 0.05 & 998 & 0.001 & 0.00332 \\
\hline
\end{tabular}

Notes: $Q_{\mathrm{i}}$ is the volume flow rate at the inlet; $R e_{\mathrm{i}}$ is the Reynolds number at the inlet; $R$ is the split ratio $\left(Q_{\mathrm{o}} / Q_{\mathrm{i}}\right) ; \mu$ is the viscosity. 
enforcing the geometric conservation constraints $\left(\sum \alpha^{(k)}=1\right)$ (Darwish et al, 2001). The Carver procedure (Carver, 1982) is also employed for bounding the volume fractions between 0 and 1.

In order to calculate the convection and diffusion terms, high resolution SMART within the context of NVSF methodology (Darwish and Moukalled, 1994) and second order central difference scheme are used respectively. All of the simulations are performed in unsteady mode using implicit three time level (TTL) method (Ferziger and Peric, 2002). The time steps were changed from $10^{-3}$ to $5 \times 10^{-5}$ during the convergence sequence.

The simulations are started with single phase $k-\varepsilon$ model and after preliminary convergence, switched to the RSMLRR model. The converged solution of single phase flow is considered as an initial condition of two phase flow. The total integration time needed to obtain a steady state result is about 1.2 seconds.

The convergence is assessed by comparing the normalized sum of absolute residuals over all control volumes with some reference value. The residual in a convergence state is in the order of $10^{-4}$ for continuity and $10^{-5}$ for all other equations.

The under relaxation factors are assumed as 0.2-0.4 for pressure equation, 0.3-0.5 for momentum equations, $0.2-$ 0.5 for volume fraction equations and 0.1-0.5 for turbulent equations. The larger values for under relaxations are implemented at the start of simulations.

\section{Results and discussion}

\subsection{Velocity distribution}

Fig. 4(a) depicts a comparison between simulated single phase tangential velocity for case 2 at different axial positions inside the hydrocyclone and velocities measured by Bai et al (2009). The numerical and experimental results are in good agreement. Small deviations can be seen for the location of maximum tangential velocity. Maximum tangential velocity occurs near the axis of hydrocyclone and decreases towards the wall. Tangential velocity distributions for case 1 (Colman's design) are also shown in Fig. 5(a). Tangential velocity has a shape of Rankine vortex, i.e. forced vortex near the axis of rotation and free vortex in outer region. This is also reported by other researchers (Hargreaves and Silvester, 1990; Bai et al, 2009). The width of free and forced regions is different between the two designs and is highly dependent on swirl intensity distribution along the hydrocyclone axis.

Fig. 4(b) shows the radial variation of axial velocity of the single phase in case 2 at different axial positions for a split ratio of $5 \%$. The experimental measurements and simulated results are in good agreement with each other. The axial velocity profiles for case 1 are shown in Fig. 5(b). The outer vortex moves downward to the underflow of hydrocyclone and the inner vortex moves upward toward the over flow.

The recirculation zones in case 1 are stronger than that in case 2 . The maximum axial distance that has negative upward velocity occurs at the location of $1,000 \mathrm{~mm}$ and $210 \mathrm{~mm}$ in case 1 and 2, respectively. The negative upward velocity affects the distribution of oil in case 1 compared with case 2 .

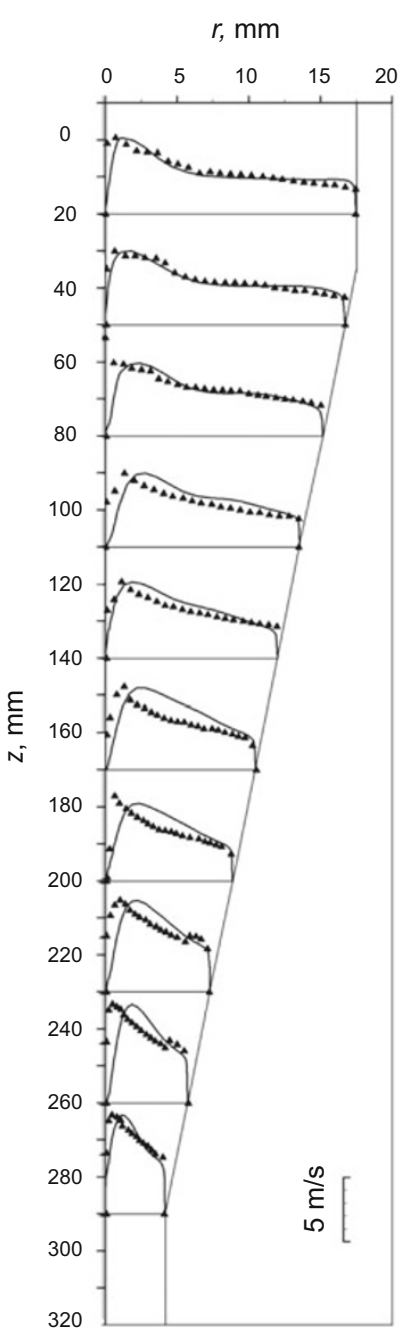

(a) Tangential velocity

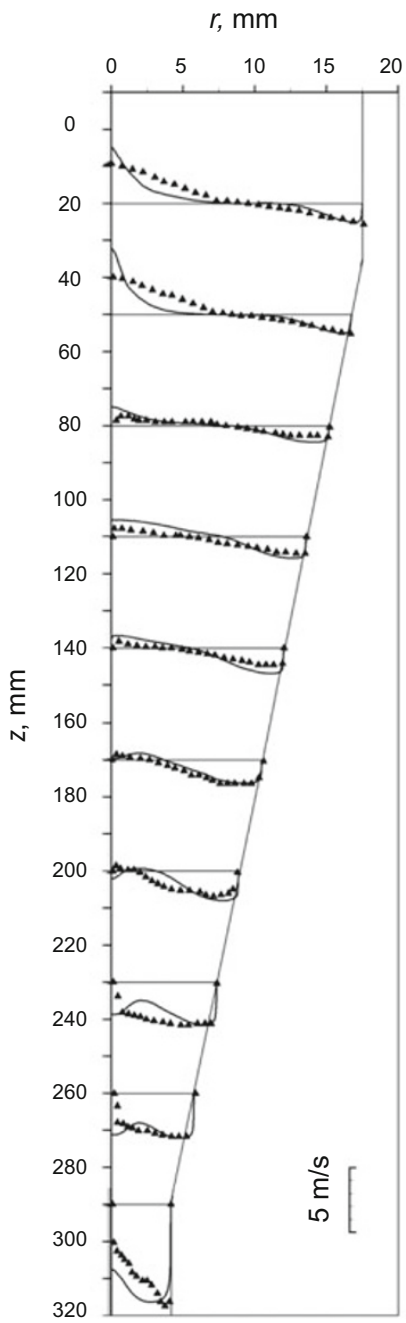

(b) Axial velocity
Fig. 4 Comparison of velocity distribution between experimental data $(\mathbf{\Delta})$ (Bai et al, 2009) and results of numerical simulations (-) for case 2

\subsection{Separation efficiency}

Separation efficiency of the standard Colman's design (case 1) is compared with available experiments (Young et al, 1994) in Fig. 6. It can be seen that numerical simulations can predict separation efficiency curve adequately. A slight over prediction of separation efficiency has occurred because of two reasons:

1) In numerical simulation, only the median diameter of droplets is used for calculating the inter-phase forces. Also it is assumed in Eq. (4) that all droplets have the same diameter. However, droplets with various diameters, either larger or smaller than median, are found in experimental measurements. Separation efficiency of smaller diameter oil droplets is less than larger ones. Therefore, the efficiency of the hydrocyclones is over predicted in numerical simulations.

2) The wall shear stress and pressure drop in numerical simulations are smaller than the real operating condition and the swirling flow maintains its swirl motion for a longer axial distance. So the location of negative axial velocity occurs in a longer axial distance in numerical simulations compared to 


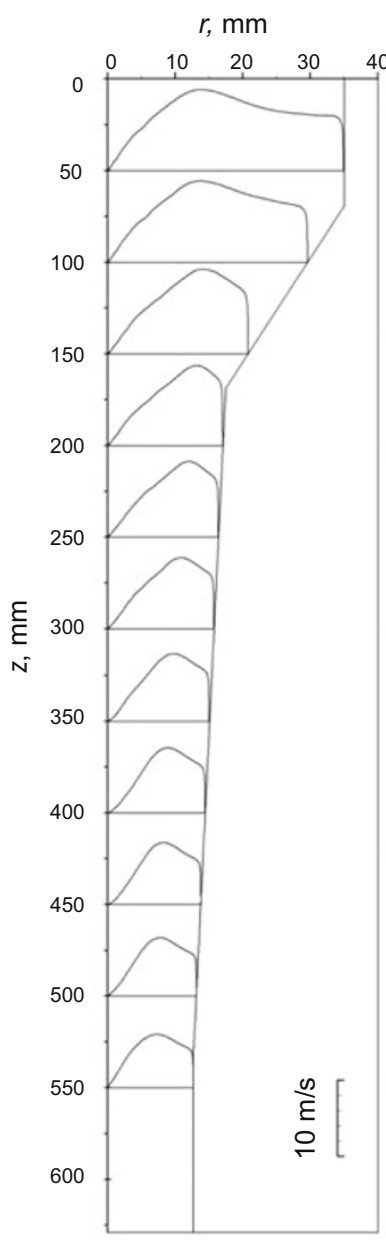

(a) Tangential velocity

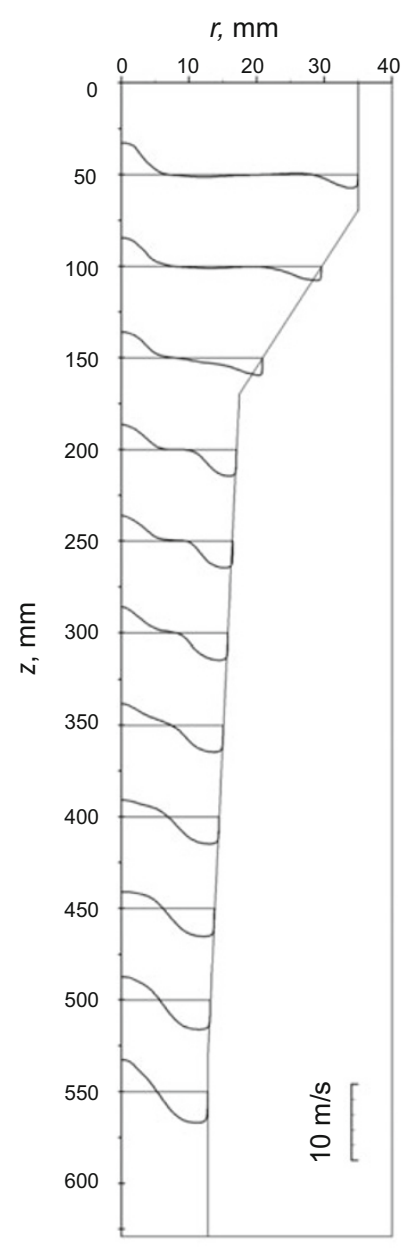

(b) Axial velocity
Fig. 5 Velocity distribution of the continuous phase inside the deoiling hydrocyclone for case 1

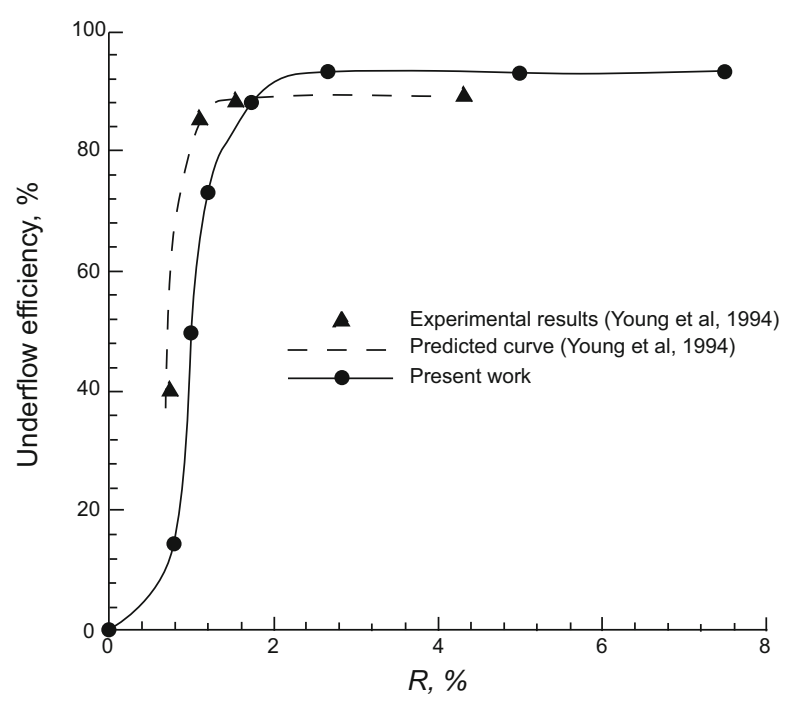

Fig. 6 Comparison of experimental (Young et al, 1994) and numerical simulation results for underflow efficiency of the deoiling hydrocyclone experimental data. Therefore, more droplets are captured by upward reverse flow.

\subsection{Oil distribution}

Distribution of oil inside hydrocyclones for both cases is shown in Fig. 7. As a result of the pressure difference in the radial direction, generated by swirling motion of the fluid, oil droplets accumulate near the axis of the hydrocyclone.

The radial distribution of oil volume fraction in three different axial distances from the top wall of hydrocyclones is shown in Fig. 8. As shown before (i.e. Figs. 4 and 5), the maximum tangential velocity in case 2 occurs closer to the axis, compared to case 1 . The high value of tangential velocity in case 2 creates a region of oil volume fraction close to 1.0 near the axis and suddenly dipped to value of 0.05 in the immediate vicinity of the axis. The peak in distribution of oil volume fraction in case 1 is not as sharp as that in case 2. Variation of the axial velocity affects the distribution of oil inside the hydrocyclone. The negative upward velocity in case 1 creates a concentration valley near the axis.

The oil concentration decreases gradually along the hydrocyclone axis for case 1 , while in case 2 , the peak in the oil volume fraction can be seen even in the axial distance of $z / D=8.0$ and it seems that the Young's design (case 2) can operate at flow split of more than $5 \%$.

It should be mentioned that previous researchers could achieve neither an accurate oil distribution inside deoiling hydrocyclones nor the separation efficiency, due to using weak multiphase models (ASM model).

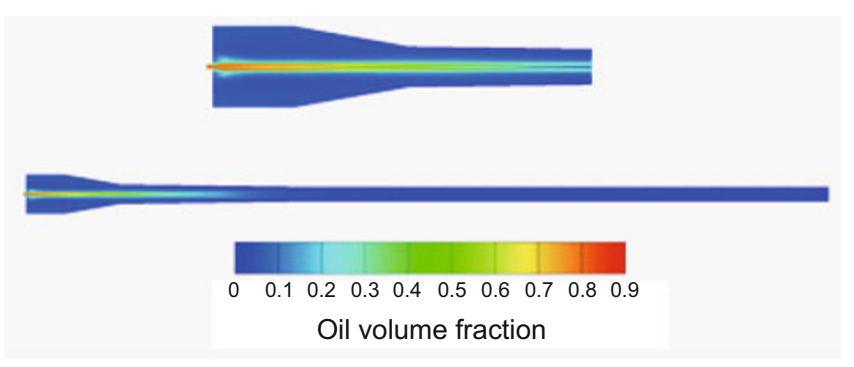

(a) Case 1

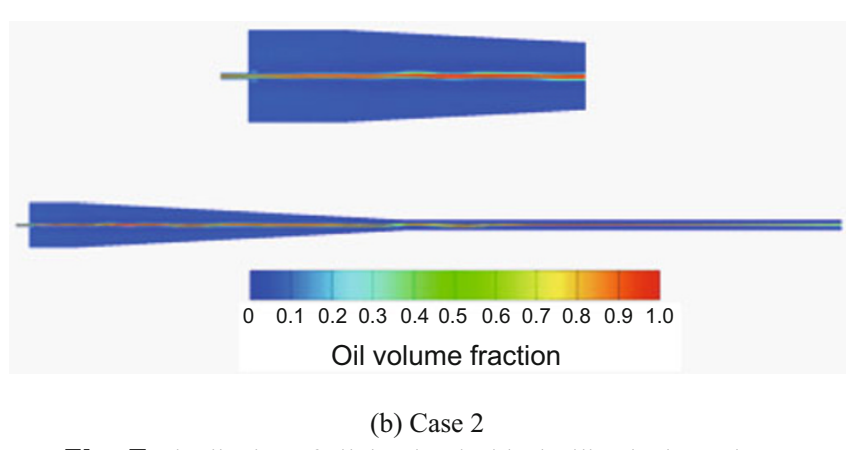

Fig. 7 Distribution of oil droplets inside deoiling hydrocyclones

\subsection{Swirl decay rate}

As discussed previously, the radial pressure gradient generated by swirling flow makes the lighter phase migrate toward the center. The migration velocity, also called slip velocity, is a function of density difference between dispersed 


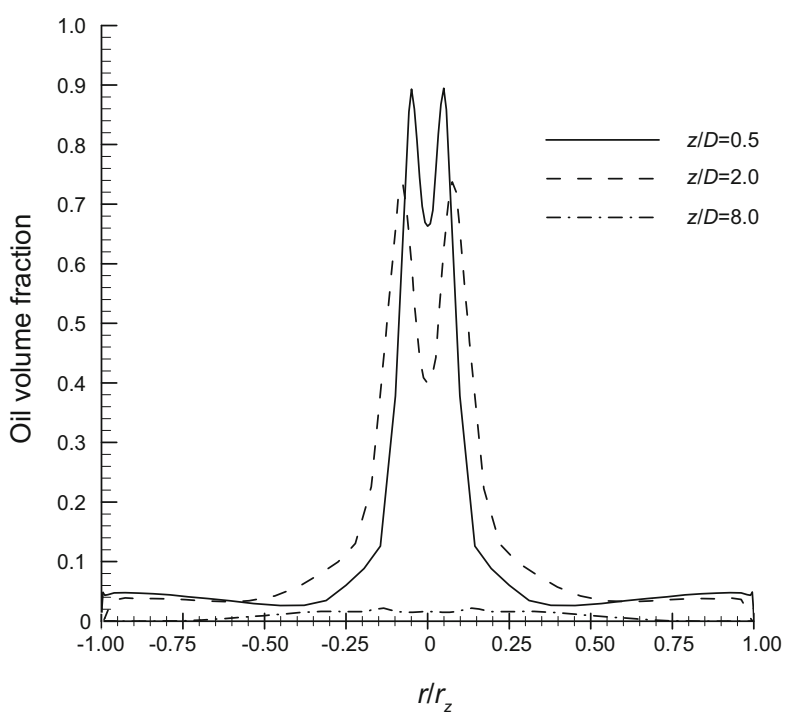

(a) Case 1

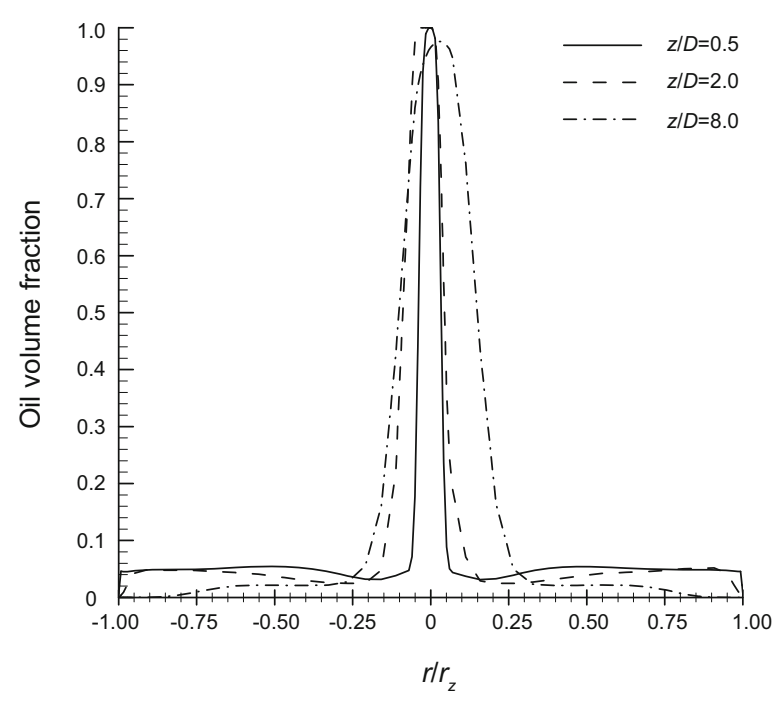

(b) Case 2

Fig. 8 Radial distribution of the volume fraction of the secondary phase (oil) at different axial positions from the top wall

and continuous phase, radial pressure gradient, relaxation time of droplets and the residence time of flow (Wolbert et al, 1995). If the migration velocity is great enough so that the oil droplets arrive at locus of zero axial velocity before the bulk flow leaves the hydrocyclone, oil droplets can be separated (Wolbert et al, 1995).

Hydrocyclone designers try to increase the flow residence time by increasing the length to diameter ratio of deoiling hydrocyclones. On the other hand, the effects of wall shear stress and stream-wise friction reduce the spinning motion of flow. Therefore, in order to have a reasonable value of tangential velocity, the cross sectional area should be reduced along the axis. An increase in the tangential velocity is proportional to the inverse of radius, due to conservation of angular momentum, while the axial velocity is proportional to the inverse of the radius squared, due to conservation of mass. Therefore, the area reduction decreases residence time of the flow and separation efficiency. These effects could be investigated using swirl number, defined as the ratio of axial flux of angular momentum to the axial flux of linear momentum. Many researchers tried to relate the velocity distribution and separation efficiency of deoiling hydrocyclones to the swirl number (Caldentey, 2000; Gomez, 2001).

The swirl number variation along the hydrocyclone axis for both cases is shown in Fig. 9. The change in the cone angle of hydrocyclones results in the slope variation of the swirl decay rate.

As a result of higher inlet flow rate, the inlet swirl number of case 1, i.e. Colman's design, is greater than that of case 2 . The swirl number in case 1 , swiftly declines in the first conical section and continues to decrease gradually in the second conical and straight following sections. The swirl decay rate in the Young's design, case 2, is not as steep as in Colman's one, case 1, and the flow can keep its swirling motion in the half of the hydrocyclone length.

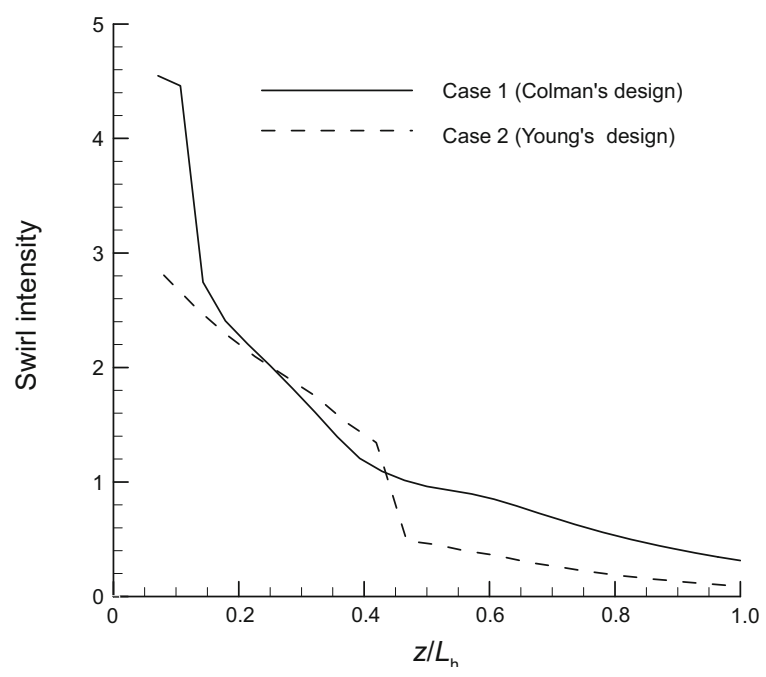

Fig. 9 Swirl intensity decay rate for different configurations of deoiling hydrocyclones

As a result of dissimilar swirl distribution between cases 1 and 2 , the difference can be seen not only in velocities, both axial and tangential, but also in oil distribution inside deoiling hydrocyclones. Therefore, it seems that the design of the swirl chamber should be according to the amount of the swirl rate needed for proper separation. Designing a swirl chamber with appropriate swirl distribution is imperative in achieving an efficient deoiling hydrocyclone.

\section{Conclusions}

Velocity and oil distributions inside deoiling hydrocyclones are obtained using a general code based on the Eulerian-Eulerian multiphase model. The turbulent stresses are approximated using the mixture approach of Reynolds stress model. The results of velocity distribution are validated 
using experimental data and showed that the RSM model is an appropriate choice for modeling multiphase flow inside deoiling hydrocyclones. A slight over prediction is also seen in the results of separation efficiency due to the fact that the median diameter of oil droplets is considered for the estimation of the drag coefficient.

The distributions of oil droplets in various axial distances from the top wall of the hydrocyclone for two different designs are compared and it is shown that the swirl intensity distribution inside the hydrocyclone can affect the velocity fields and oil distribution. Furthermore, the idea of designing efficient hydrocyclones based on appropriate swirl intensity distribution is introduced, which will be addressed in the development of present work.

\section{Acknowledgements}

The authors would like to thank Professor Marwan Darwish, American University of Beirut/Mechanical Engineering Department, for his guidance and help in developing the multiphase part of this research.

\section{References}

Bai Z, Wang H and Tu S. Experimental study of flow patterns in deoiling hydrocyclone. Miner. Eng. 2009. 22(4): 319-323

Belaidi A and Thew M T. The effect of oil and gas content on the controllability and separation in a de-oiling hydrocyclone. Chem. Eng. Res. Des. 2003. 81(3): 305-314

Caldentey J C. A Mechanistic Model for Liquid Hydrocyclones (LHC). MS Thesis. The University of Tulsa. 2000

Carver M B. A method of limiting intermediate values of volume fraction in iterative two-fluid computations. J. Mech. Eng. Sci. 1982. 24: $221-224$

Choi M S. Hydrocyclone produced water treatment for offshore developments. Paper SPE 20662 presented at 65th Annual Technical Conference and Exhibition of the Society of Petroleum Engineers, 1990, New Orleans

Colman D. The Hydrocyclone for Separating Light Dispersions. Ph.D. Thesis. Southampton University, UK. 1981

Colman D and Thew M. Hydrocyclone to give a highly concentrated sample of a lighter dispersed phase. Presented at International Conference on Hydrocyclones, 1980, BHRA, Cambridge, United Kingdom 15: 209-223

Colman D and Thew M. Correlation of separation results from light dispersion hydrocyclones. Chem. Eng. Res. Des. 1983. 61: 233-240

Colman D and Thew M. Cyclone Separator. U.S. Patent: 4764287. 1988

Colman D, Thew M and Corney D. Hydrocyclones for oil/water separation. Presented at International Conference on Hydrocyclones, 1980, BHRA, Cambridge, United Kingdom, 11: 143-165

Darwish $\mathrm{M}$ and Moukalled F. Normalized variable and space formulation methodology for high-resolution schemes. Numer. Heat Transfer B. 1994. 26(1): 79-96.

Darwish M, Moukalled F and Sekar B. A unified formulation of the segregated class of algorithms for multifluid flow at all speeds. Numer. Heat Transfer Part B. 2001. 40(2): 99-137

Davidson L and Farhanieh B. Manual of CALC-BFC. Chalmers University of Technology, Gothenburg, Sweden. 1991

Ditria J C and Hoyack M E. The separation of solids and liquids with hydrocyclone based technology for water and crude processing. Paper SPE 28815 presented at SPE Asia and Pacific Oil \& Gas Conference, 1994, Melbourne
Drew D A. Mathematical modeling of two-phase flow. Ann. Rev. Fluid Mech. 1983. 15: 261-91

Enwald H, Peirano E and Almstedt A E. Eulerian two-phase flow theory applied to fluidization. International Journalof Multiphase Flow. 1996. 22(1): 21-66

Falnigan D A, Stolhand J E, Shimoda E, et al. Use of low-shear pumps and hydrocyclones for improved performance in the cleanup of lowpressure water. Paper SPE 19743 presented at SPE Annual Technical Conference and Exhibition, 1992, San Antonio

Ferziger J H and Peric M. Computational Methods for Fluid Dynamics. Springer-Verlag Berlin Heidelberg. 2002

Gomez C H. Oil-water separation in liquid-liquid hydrocyclones (LLHC): Experiment and modeling. MS Thesis. The University of Tulsa. 2001

Grady S A, Wesson G D, Abdullah M, et al. Prediction of 10-mm hydrocyclone separation efficiency using computational fluid dynamics. Filtr. Sep. 2003. 40(9): 41-46

Hargreaves J H and Silvester R S. Computational fluid dynamics applied to the analysis of deoiling hydrocyclone performance. Chem. Eng. Res. Des. 1990. 68(4): 365-383

Huang S. Numerical simulation of oil-water numerical simulation of oil-water hydrocyclone using Reynolds-stress model for Eulerian multiphase flows. Can. J. Chem. Eng. 2005. 83(5): 829-834

Husveg T, Rambeau O, Drengstig T, et al. Performance of a deoiling hydrocyclone during variable flow rates. Miner. Eng. 2007. 20(4): 368-379

Ishii M. Thermo-fluid Dynamic Theory of Two-Phase Flow. 1975. Paris: Eyrolles

Jones P S. A field comparison of static and dynamic hydrocyclones. Paper SPE 20701 presented at SPE Annual Technical Conference and Exhibition, 1993, New Orleans

Kharoua N, Khezzar L and Nemouchi Z. Hydrocyclones for de-oiling applications-A review. Pet. Sci. Tech. 2010. 28(7): 738-755

Launder B E, Reece G J and Rodi W. Progress in the development of a Reynolds-stress turbulence closure. J. Fluid Mech. 1975. 68: 537566

Lien F S and Leschziner M A. Assessment of turbulence-transport models including non-linear RNG eddy-viscosity formulation and second-moment closure for flow over a backward-facing step. Comput. Fluids. 1994. 23(8): 983-1004

Meldrum N. Hydrocyclones: A solution to produced-water treatment. Paper SPE 16642 presented at 19th Annual Conference on Offshore Technology, 1988, Houston

Moukalled F and Darwish M. Pressure-based algorithms for multifluid flow at all speeds - Part I: Mass conservation formulation. Numer. Heat Transfer Part B. 2004. 45(6): 495-522

Noort F J, Etten J P and Donders R S. Reduction of residual oil content in produced water at offshore gas production platform P/6A. Paper SPE 20882 presented at Europe's 90 Conference, 1990, Netherlands

Noroozi S and Hashemabadi S H. CFD simulation of inlet design effect on deoiling hydrocyclone separation efficiency. Chem. Eng. Technol. 2009. 32(12): 1885-1893

Noroozi S and Hashemabadi S H. CFD analysis of inlet chamber body profile effects on de-oiling hydrocyclone efficiency. Chem. Eng. Res. Des. 2011. 8(7): 968-977

Petty C A and Parks S M. Flow structures within miniature hydrocyclones. Miner. Eng. 2004. 17(5): 615-624

Rhie C M and Chow L W. Numerical study of the turbulent flow past an airfoil with trailing edge separation. AIAA J. 1983. 21(11): 15271532

Saboni A and Alexandrova S. Numerical study of the drag on a fluid sphere. AIChE J. 2002. 48(12): 2992-2994

Sheng H P, Welker J R and Sliepcevich C M. Liquid-liquid separation in 
a conventional hydrocyclone. Can. J. Chem. Eng. 1974. 52(4): 487491

Simkin D J and Olney R B. Phase separation and mass transfer in a liquid-liquid cyclone. AIChE J. 1956. 2(4): 545-551

Thew M T. Hydrocyclone redesign for liquid-liquid separation. The Chem. Engineer. 1986. July/August: 17-23

Van den Broek W M G T and Plat R. Characteristics and possibilities of some techniques for de-oiling of production water. Paper SPE 23315 presented at First International Conference on Health, Safety and Environmental. 1991. Netherlands

Van den Broek W M G T, Plat R and Van der Zande M J. Comparison of plate separator, centrifuge and hydrocyclone. Paper SPE 48870 presented at SPE International Conference and Exhibition, 1998, Beijing
Wilcox C D. Turbulence Modeling for CFD. 1994. DCW Industries Inc. Wolbert D, Ma B F, Aurelle Y, et al. Efficiency estimation of liquidliquid hydrocyclones using trajectory analysis. AIchE J. 1995. 41(6): 1395-1402

Young G A B, Wakley W D, Taggart D L, et al. Oil-water separation using hydrocyclones: An experimental search for optimum dimensions. J. Pet. Sci. Eng. 1994. 11(1): 37-50

Zhou N, Gao Y, An W, et al. Investigation of velocity field and oil distribution in an oil-water hydrocyclone using a particle dynamics analyzer. Chem. Eng. J. 2009. 157(1): 73-79

Zuber N. On the dispersed two phase flow in the laminar regime. Chem. Eng. Sci. 1964. 19(11): 897-917

(Edited by Sun Yanhua) 